\title{
Biocleaning of starch glues from textiles by means of $\alpha$-amylase- based treatments
}

\author{
Mariagrazia Tortora ${ }^{1}$, Francesca Gherardi ${ }^{1 *}$, Enrico Ferrari ${ }^{2}$, and Belinda Colston ${ }^{1 *}$ \\ ${ }^{1}$ School of Chemistry, University of Lincoln, Lincoln LN6 7TS, UK; \\ Email and ORCID number: mtortora@lincoln.ac.uk, 0000-0003-2149-3775 \\ fra.gherardi@gmail.com, 0000-0003-0469-8154 \\ bcolston@lincoln.ac.uk,0000-0003-4457-0323 \\ ${ }^{2}$ School of Life Sciences, University of Lincoln, Lincoln LN6 7TS, UK; \\ Email and ORCID number: eferrari@lincoln.ac.uk, 0000-0002-9102-7422 \\ Corresponding authors: fra.gherardi@gmail.com \\ bcolston@lincoln.ac.uk
}

\begin{abstract}
Glues based on starch are widely used for the consolidation of brittle fibres in historic and archaeological textiles. Aging fabrics are affected by hydrolysis/oxidation and cross-linking of these glues, a decrease of glues' solubility, the formation of cracks, and discoloration. The hydrolytic action of enzymes on starch-based glues is promising, as molecular recognition offers great selectivity. However, a systematic assessment of the best methods for applying enzymatic formulations has not been explored yet. Here, $\alpha$-amylase was applied either by pipetting a solution or combining with gellan gel (embedded in the gel or spread on the gel surface). The effectiveness of the different formulations on the removal of potato and wheat starch was evaluated by Fourier Transform Infrared (FTIR) spectroscopy, Scanning Electron Microscopy (SEM) and colorimetric measurements. Enzymes dispersed in gel showed weak diffusion at the surface, resulting in poor starch breakdown and removal. On the contrary, amylase applied by pipette and spread on gel resulted in high starch removal selectivity and efficiency, with neither swelling nor damage to the fibres. These results validate protocols for the assessment of the enzymatic activity on glue-consolidated fibres, identify best application methods and confirm the excellent properties of amylase dispersions for the conservation of historic and archaeological textiles.
\end{abstract}

\section{Key points}

1. Application of $\alpha$-amylase by pipette and combined with gellan gel to remove starch glues from wool.

2. Systematic assessment of the best application methods following a multi-analytical protocol.

3. Enzymes dispersed in gel exhibit poor diffusion at the surface, leading to weak starch removal.

4. Enzymes applied by pipette and spread on gel are efficient in starch cleaning, without damage to the fibres.

Keywords: $\alpha$-amylase; starch cleaning; cotton; wool; gels; FTIR spectroscopy 


\section{Introduction}

Glues based on starch, collagen or casein have been widely used to consolidate brittle fibres in historic and archaeological textiles. However, these glues tend to undergo hydrolysis/oxidation and crosslinking reactions, becoming insoluble and leading to discoloration, biodeterioration, and formation of cracks and tensions in the fibres (Mazzoli et al. 2018). For the preservation of textile artefacts, therefore, it is essential to remove completely aged consolidants and glues. In this context, the hydrolytic action of enzymes is very promising, as they exhibit great selectivity in the cleaning of unwanted materials, without compromising the historic and artistic substrates (Bosch-Roig and Ranalli 2014; Cremonesi 2013; Decoux 2002; Mazzoli et al. 2018; Palla and Barresi 2017). Due to their negligible toxicity, their use as substitutes of organic solvents is widely reported on different substrates, such as wall paintings (Beutel et al. 2002; Ranalli et al. 2005), paintings on canvas (Pereira et al. 2013), paper (Decoux 2002; Mazzuca et al. 2014, 2017; Schwarz et al. 1999), textiles (Ahmed and Kolisis 2011; Bott 1990; Chapman 1986; Ferrari et al. 2017; Shibayama and Eastop 1996), stones (Valentini et al. 2010, 2012), and acrylic-based graffiti and coatings (Bellucci et al. 1999; Germinario et al. 2017). In particular, amylases, which catalyze starch hydrolysis, enable adequate removal of starch films with relatively shorter times compared to those required following traditional methods, like rinsing and humidification (Schwarz et al. 1999). Starch is a polysaccharide made of two components, amylose and amylopectin, comprising glucose monomers joined by glycosidic bonds. Two types of amylases can breakdown starch by catalyzing the hydrolysis of $\alpha-1,4$ glycosidic bonds. $\alpha$-amylase is an endo-enzyme, which causes random hydrolysis of glycosidic bonds and depolymerizes both amylose and amylopectin. $\beta$-amylase is an exo-enzyme, which only works on terminal glucose residues of amylose or amylopectin and produces maltose and $\beta$-limit dextrin (van der Maarel et al. 2002). The random fragmentation of amylose and amylopectin by $\alpha$-amylase leads to a rapid reduction in viscosity and to a fast increase in water solubility, which makes starch easily removable from surfaces (Gupta et al. 2003; van der Maarel et al. 2002). Thus, $\alpha$-amylase water solutions have been adopted for the removal of starch substrates, thanks to their ability to spread and adsorb on the solid surface (Božić et al. 2017). Unfortunately, the use of large volumes of water to apply the enzymatic formulations and to remove the digestion residues can cause swelling of fabrics and solubilization of dyes. Enzyme immobilization, besides reducing the volume of water required, presents more convenient handling, and enhancement of enzyme stability (Gherardi et al. 2019; Sheldon 2007). It is now common practice to use poultices and gels as supports in conservation interventions (Baglioni et al. 2014). Among these, gellan-gum hydrogel recently received particular attention, due to its stability, rigidity, transparency, and for its easy preparation. Different systems have been explored to apply enzymatic solutions, i.e. by dispersing them in gellan gel (Mazzuca et al. 2014) or by spreading them on the gel (Ferrari et al. 2017). Despite the extensive use of $\alpha$-amylase in conservation, a systematic study of its efficacy in starch hydrolysis depending on the method of application has not been published yet.

This research investigates the effectiveness of $\alpha$-amylase solutions on the cleaning of potato and wheat starch-coated wool samples, by testing different application methods: by pipette, embedded in gellan gel, and spread on gel.

\section{Materials and Methods}

Preparation of $\alpha$-amylase dispersion: Type II-A from Bacillus sp. (A6380, Sigma-Aldrich, Dorset, United Kingdom) was dissolved in a buffer solution ( $20 \mathrm{mM}$ HEPES pH=7.3, $100 \mathrm{mM} \mathrm{NaCl}$ ) at a concentration of $3 \mathrm{mg} / \mathrm{mL}(60 \mu \mathrm{M})$. The enzyme has an estimated molecular weight of 50-55 kDa by SDS-PAGE and $\geq 1,500$ units/mg protein (biuret) according to the technical data sheet. 
Evaluation of the enzymatic activity: Fourier Transform Infrared (FTIR) spectroscopy studies were performed on PerkinElmer (Waltham, United States) Spectrum 100 FTIR Spectrometer equipped with a deuterated triglycine sulfate (DTGS) detector. Dispersions of $1 \%$ and $2 \%(\mathrm{w} / \mathrm{v})$ of potato (Sigma Aldrich, Dorset, United Kingdom) and wheat starch (Kremer Pigmente, Aichstetten, Germany) boiled in water with stirring for 30 minutes were applied as thin film on silicon polished windows (Crystran Ltd, Poole, United Kingdom). Following $120 \mathrm{~min}$ and $240 \mathrm{~min}$ of exposure to enzymatic dispersions, FTIR spectra were acquired (64 scans, resolution $4 \mathrm{~cm}^{-1}$ ). Background spectra were recorded with silicon windows and subtracted from the sample spectra. All spectra were normalized on the intensity of the $\mathrm{C}-\mathrm{H}$ stretching vibration at $2930 \mathrm{~cm}^{-1}$. The starch hydrolysis $(\mathrm{SH}, \%)$ was calculated using the following equation: $S H=\left(R_{f}-R_{i}\right) / R_{i} \times 100$, where $R_{i}$ and $R_{f}$ are the intensity ratios of the $1045 / 1022 \mathrm{~cm}^{-1}$ peaks before and after treatment with the enzymatic dispersions. Another set of silicon windows covered with $1 \%$ and $2 \%(\mathrm{w} / \mathrm{v})$ of potato and wheat starch was thermally aged in an oven for 10 days at $80^{\circ} \mathrm{C}$ to simulate the chemical-physical properties of naturally aged starch. The starch hydrolysis was then evaluated after 120 and $240 \mathrm{~min}$ of exposure to amylase. Each experiment was repeated three times.

Aiming at identifying the most appropriate application procedure in textile conservation, the amylase dispersions were applied on wool specimens coated with $1 \%, 2 \%$ and $5 \%(\mathrm{w} / \mathrm{v})$ potato and wheat starch (labelled as P1, P2, P5 and W1, W2, W5, respectively) using the three application methods. Before assessing the effectiveness of amylase, starch-coated wool samples were thermally aged in an oven for 10 days at $80^{\circ} \mathrm{C}$, to simulate physico-chemical conditions of naturally aged starch glues. An aliquot of $2 \mu \mathrm{L}$ amylase dispersion was applied on wool samples by pipette and by using $2 \%(\mathrm{w} / \mathrm{v})$ gellan gum (Gellan Kelcogel, CTS, Vicenza, Italy) in deionized water, to achieve a slower release of amylase to the textile. In particular, $0.5 \mathrm{~cm}$ thick amylase-loaded gels were prepared following 2 methods. For the 'amylase in gel' method, the same volume of $\alpha$-amylase solution was added to the gellan gels $\left(\sim 1.5 \mu \mathrm{L} / \mathrm{cm}^{2}\right)$, at about $60^{\circ} \mathrm{C}$ during cooling in a Petri dish. Instead, for the 'amylase on gel' method the amylase dispersion was applied by pipette on the solid gel surface in order to localize amylase at the interface of the textile and the gel. Amylase immobilized on gellan gels were then applied on wool samples for 120 min. Starch digestion by amylase dispersions was monitored by FTIR spectroscopy and an Attenuated Total Reflection (ATR) diamond crystal accessory. All spectra were normalized on the intensity of the $\mathrm{C}=\mathrm{O}$ stretching vibration of amide I band at $1650 \mathrm{~cm}^{-1}$. Finally, the surface was treated with a cotton swab soaked with deionized water in order to remove the enzymatic formulations and the residues of starch digestions. ATR-FTIR measurements were repeated to assess the complete removal of amylase. Each result was calculated by averaging three individual experiments.

Stereomicroscopy and colour measurements on dyed and undyed wool fibres coated with potato and wheat starch: stereomicroscopy observations and colour measurements were carried out to evaluate the removal of starch from thermally-aged wool samples by using a Zeiss Stereo Discovery V8 stereomicroscope (Oberkochen, Germany) and Konica Minolta (Tokyo, Japan) CR-410 Chroma Meter instrument with a D65 illumination. Measurements were processed according to the CIE L*a*b* standard colour system. Five measurements were performed on each area (about $4 \mathrm{~cm}^{2}$ ) and the average results of $L^{*} a^{*} b^{*}$ were used to calculate the colour difference $\Delta E_{2,1}$ between treated and untreated areas: $\left.\Delta E_{2,1}=\left[\left(L{ }_{2}-L{ }_{1}\right)^{2}+\left(a^{*}{ }_{2}-a{ }_{1}\right)^{2}+\left(b^{*}{ }_{2}-b{ }^{*}\right)^{2}\right]^{1 / 2}\right)$. The experiment was carried out on undyed samples and wool dyed with natural dyes (madder, indigo and saffron).

SEM-EDS on wool samples coated with potato and wheat starch: thermally-aged wool samples with $1 \%, 2 \%$ and $5 \%(\mathrm{w} / \mathrm{v})$ potato and wheat starch were observed in secondary electrons mode before and 
after the application and final washing of the amylase dispersions by means of Scanning Electron Microscopy (SEM) (Inspect S; FEI Inc., Altrincham, United Kingdom) and Energy Dispersive X-ray (EDS) detector (Inca X-ray spectrometer; Oxford Instruments Ltd., Abingdon-on-Thames, United Kingdom). The samples were mounted on aluminium stubs and sputtered with a gold layer of $10 \mathrm{~nm}$. The SEM operated at an acceleration voltage of $1.5 \mathrm{kV}$.

\section{Results}

To examine the activity of $\alpha$-amylase in the hydrolysis of both unaged and thermally-aged starch films, $1 \%$ and $2 \% \mathrm{w} / \mathrm{v}$ potato and wheat starches were applied on silicon windows and the modifications of their conformation were monitored by FTIR spectroscopy after 120 and 240 min of digestion (Fig. 1). Changes in the spectral region of $950-1100 \mathrm{~cm}^{-1}$ ascribed to $\mathrm{C}-\mathrm{O}$ and $\mathrm{C}-\mathrm{C}$ stretching vibrational modes were studied (Bello-Pérez et al. 2005; Gherardi et al. 2019; Goodfellow and Wilson 1990; Htoon et al. 2009; Lopez-Rubio et al. 2008; van Soest et al. 1995), with a focus on the bands at $1022 \mathrm{~cm}^{-1}$ and 1045 $\mathrm{cm}^{-1}$ which are associated with amorphous and ordered/crystalline regions in starch, respectively (Bello-Pérez et al. 2005; Htoon et al. 2009; van Soest et al. 1995). After digestion by amylase, a higher ratio of 1045/1022 cm $\mathrm{cm}^{-1}$ peak intensities occurs, due to the decrease of the length of the starch chain and increase of the crystallinity (Gherardi et al. 2019; Lopez-Rubio et al. 2008; Palacios et al. 2004). In Fig. 1 we observed a general reduction of the intensities of the absorption bands and an increase in the $1045 / 1022 \mathrm{~cm}^{-1}$ peak ratio after the application of the enzymatic dispersions. The percentage of increase of crystallinity was used to calculate SH values (Fig. 1e) and no meaningful SH increase was observed after 120 minutes exposure. For unaged starch, the lower digestion of $\mathrm{P} 2$ and W2 compared to P1 and W1 (starch hydrolysis values after 240 minutes exposure were about $37 \%$ and $15 \%$ for P1 and $\mathrm{P} 2$ and $41 \%$ and $26 \%$ for $\mathrm{W} 1$ and $\mathrm{W} 2$, respectively) can be related to reduced ability of amylase to reach and orient its active sites towards more concentrated substrates (Fig. 1e).

After thermal ageing of potato and wheat starch films, their FTIR spectra showed a higher 1045/1022 $\mathrm{cm}^{-1}$ peak ratio, especially for $\mathrm{P} 1$ and $\mathrm{W} 1$, due to an increase of crystallinity (Supporting Information, Fig. S1). Lower efficacy in starch digestion by amylase was achieved on thermally-aged starches, especially P1 and W1 (SH values after 240 minutes exposure were about $17 \%$ and $7 \%$ for $\mathrm{P} 1$ and $\mathrm{W} 1$, respectively) (Fig. $1 \mathrm{e}$ ), due to the fact that the increase of ordered regions in starch makes them more resistant to the hydrolysis by amylase (Abduh et al. 2019).

To determine which application method of the enzymatic dispersions leads to the most effective breakdown and removal of starch, ATR-FTIR spectroscopy was used on thermally-aged wool samples coated with wheat and potato starch. The spectra in Fig. 2 and 3 show the characteristic absorption bands of protein: amide I band at $1650 \mathrm{~cm}^{-1}$ (C=O stretching vibration), amide II at about $1540 \mathrm{~cm}^{-1}(\mathrm{~N}-$ $\mathrm{H}$ bending/C-N stretching vibrations) and amide III at about $1230 \mathrm{~cm}^{-1}$ ( $\mathrm{N}-\mathrm{H}$ bending/C-N stretching vibrations). The amylase dispersion was applied following three methods: pipette, in gel and on gel. Different results were achieved, highlighting dissimilar interaction of amylase with the substrate. When enzymes were applied by pipette and on gel (Fig. 2 and 3 a and e, b and f), a significant increase of the ratio of 1045/1022 cm-1 peak intensities was observed. In particular, amylase applied by pipette and on gel was particularly effective in the hydrolysis of P2 and W2 starch, respectively (Fig. 2). Similar results were obtained on wool samples coated with P5 and W5 starches (Fig. 3). In both potato and wheat starch-coated wool samples, the introduction of amylase dispersion in gellan gel was not effective in promoting starch hydrolysis, as evidenced by FTIR spectra, which exhibit almost no increase in the ratio of $1045 / 1022 \mathrm{~cm}^{-1}$ peak intensities (Fig. 2c and 3c). The low effectiveness of this application method is probably due to the poor ability of the gel to release enough enzymes to breakdown the more crystalline structure of potato starch. On the contrary, when immobilized on gel, 
amylase is confined on the wool surface and it can easily diffuse, promoting the effective removal of starch. ATR-FTIR spectra collected after enzymatic digestion and cleaning of residues of starch and amylase highlighted the complete removal of potato and wheat starches from wool treated with amylase applied by pipette and on gel, while starch granules were still present on the surface of wool treated with amylase immobilized in gel (Supporting Information Fig. S2 and S3).

The evaluation of the cleaning effectiveness in the removal of starch from wool samples was further supported by observations of textiles by SEM. On samples treated with $2 \% \mathrm{w} / \mathrm{v}$ (Supporting Information Fig. S4) and 5\% w/v (Fig. 4) potato and wheat starches, the application of amylase by pipette and immobilized on gellan gels resulted in complete removal of starch. On the contrary, residues of starch could be observed on samples treated with amylase dispersed inside the gel, especially on wool coated with P5 and W5 (Fig. 4).

Color measurements were carried out on aged undyed and dyed wool coated with potato and wheat starches before and after the treatment with the amylase dispersions. Starch applied on undyed wool did not significantly affect the surface color and for this reason, the three application methods of amylase were equally effective in terms of color change (Supporting Information Table S1 and Fig. S5). However, once applied on dyed wool, starch induced a higher color change and it was possible to discriminate better the effectiveness of the enzymatic dispersions. In particular, amylase applied by pipette and on gel on madder-dyed wool was able to remove the white layer of starch, with a decrease of $\mathrm{L}^{*}$ and increase of $\mathrm{b}^{*}$ coordinates, while almost no color change $\left(\Delta \mathrm{E}_{2,1}\right)$ was obtained by amylase immobilized in gel (Fig. 5 and Table S2). The inefficient starch removal by amylase in gel was also highlighted by visual and microscopic observations, as residues of starch were still visible around the fibres (Fig. 5). Similar results were achieved by the enzymatic formulations applied on wool dyed with indigo and saffron (Fig. 6 and Supporting Information Fig. S6, Fig. S7, Table S3 and S4). In particular, the effectiveness of amylase applied by pipette and immobilized on gel in the removal of potato and wheat starches was particularly evident on indigo-dyed wool, with a reduction of $\mathrm{L}^{*}$ and $\mathrm{b}^{*}$ coordinates (Fig. 6a and b and Supporting Information Fig. S7). On saffron-dyed wool, the enzymatic formulations were not able to significantly change the surface color and to reach $\mathrm{L}^{*}$, $\mathrm{a}^{*}$ and $\mathrm{b}^{*}$ values similar to those of the untreated and aged wool sample, probably due to a slight dye leaching after the application of potato and wheat starches (Fig. $6 c$ and $d$ and Supporting Information Fig. S7).

\section{Discussion}

The study by FTIR spectroscopy of the modifications in the conformations of starches applied on silicon windows provided useful information about the effectiveness of the enzymatic formulations. In particular, a significant increase of crystallinity due to starch hydrolysis was obtained within 120 minutes exposures to amylase, especially once applied on P1 and W1 (Fig. 1) (Gherardi et al. 2019; Lopez-Rubio et al. 2008). The enzymatic treatment showed lower efficacy in starch digestion of more concentrated substrates (P2 and W2) and on thermally-aged starches (Fig. 1). Indeed, denser structures resulted from more concentrated starches or induced by a thermal treatment reduce the penetration of amylase dispersion through the films, limiting the swelling and the solubility of starch (Basiak et al. 2017; Waterschoot et al. 2015).

To set-up the best application procedure, the enzymatic dispersions were applied on thermally-aged wool samples coated with wheat and potato starch, following three methods: pipette, in gel and on gel. The results obtained from ATR-FTIR spectroscopy indicate that potato and wheat starches were more easily hydrolysed and removed by amylase applied by pipette and on gel, respectively (Fig. 2 and 3). Wheat starch is characterized by higher amylose content compared to potato starch, resulting in a higher hydrophilicity and ability to absorb water (Basiak et al. 2017). This aspect can explain the reason 
why wheat starch could be hydrolysed and removed from wool samples more easily by using amylase immobilized on gel (Fig. $2 f$ and $3 f$ ), compared to potato starch. Indeed, the gel is able to release amylase and water, to slow down the drying of the enzymatic dispersion, and to promote the breakdown of starch. In addition, the high amount of crystalline regions in potato starch (especially P5) makes the film denser, reducing the diffusion of the enzymatic dispersion inside the 3D textile network, and therefore, also the starch digestion (Fig. 2 and 3) (Abduh et al. 2019; Basiak et al. 2017; Luchese et al. 2018; Waterschoot et al. 2015).

On the contrary, once incorporated inside gellan gel, amylase was not released in sufficient amount to effectively hydrolyse starch and, therefore, residues still accumulated on the surface of wool.

These results were confirmed by SEM analysis carried out on wool samples before and after the application of the enzymatic formulations. Starch clusters were found on fibres in wool samples treated with amylase dispersed in gellan gel, while a complete removal of the glues was achieved after the application of amylase by pipette and spread on gel (Fig. 4 and Supporting Information Fig. S4). The use of amylase by pipette is not always recommended for cleaning of brittle historic and archaeological textiles, as the solution can lead to the swelling of the fibres, thus compromising the artefacts (Ferrari et al. 2017). In the present study, however, neither swelling nor damage of the wool fibres occurred after the application and final washing of the enzymatic formulations.

Potato and wheat starches were applied on wool dyed with madder, saffron and indigo and microscopic observations and color measurements were carried out to monitor the complete removal of the starch-based glues. The results were in agreement with FTIR spectra and SEM observations, proving the effectiveness of amylase applied by pipette and spread on gel in the complete cleaning of starch-based glues (Fig. 5 and 6, Supporting Information Fig. S5-S7 and Table S1-S4).

The use of amylase dispersions represents a green approach for the removal of aged starch-based glues from textiles. Different application methods of the enzymatic dispersions were tested on accelerated aged wool samples coated with potato and wheat starches. The application of amylase by pipette and immobilized on gellan gel led to efficient and selective removal of starch, as it confines the enzymes on the surface of the textile, and neither swelling nor damage to the fibres were detected. In addition, starch residues were completely removed after cleaning with a cotton swab. On the contrary, by embedding amylase inside gellan gel, poor efficiency of starch digestion was observed, and residues of glues were still detected by microscopic observations, due to reduced diffusion of the enzymes through the gel. The multianalytical protocol allowed to discriminate the effectiveness of the enzymatic formulations and their application method, and the results obtained further support their use in heritage conservation, in particular for the treatment of historic textiles.

\section{Supporting Information}

Supporting characterization data (FTIR spectra of starch-digested by amylase, scanning electron microscopy and color measurements).

\section{Authors' contribution}

MT performed the analyses and drafted the manuscript. FG conceived the study, performed the analyses, carried out the interpretation of the results and wrote the manuscript. EF performed the analyses and critically reviewed the manuscript. BC supervised the activities and critically reviewed the manuscript. All authors read and approved the final manuscript. 


\section{Acknowledgements}

Dr Lyudmila Turyanska from the University of Nottingham is acknowledged and thanked for her contribution.

\section{Compliance with ethical standards \\ Funding}

The work was supported by funding from the Foundation Blanceflor Boncompagni-Ludovisi, née Bildt and the University of Lincoln.

\section{Conflicts of interest}

The authors declare no conflicts of interest and no competing financial interest.

\section{Ethical Approval}

This article does not contain any studies with human participants or animals performed by any of the authors.

\section{References}

Abduh SBM, Leong SY, Agyei D, Oey I (2019) Understanding the properties of starch in potatoes (solanum tuberosum var. Agria) after being treated with pulsed electric field processing. Foods (Basel, Switzerland) 8(5):159 doi:10.3390/foods8050159

Ahmed HE, Kolisis FN (2011) An investigation into the removal of starch paste adhesives from historical textiles by using the enzyme $\alpha$-amylase. J Cult Herit 12(2):169-179 doi:https://doi.org/10.1016/j.culher.2010.08.001

Baglioni P, Berti D, Bonini M, Carretti E, Dei L, Fratini E, Giorgi R (2014) Micelle, microemulsions, and gels for the conservation of cultural heritage. Adv Colloid Interfac 205:361-371 doi:https://doi.org/10.1016/j.cis.2013.09.008

Basiak E, Lenart A, Debeaufort F (2017) Effect of starch type on the physico-chemical properties of edible films. Int J Biol Macromol 98:348-356 doi:https://doi.org/10.1016/j.ijbiomac.2017.01.122

Bello-Pérez LA, Ottenhof MA, Agama-Acevedo E, Farhat IA (2005) Effect of storage time on the rtrogradation of banana starch extrudate. J Agr Food Chem 53(4):1081-1086 doi:10.1021/jf048858I

Bellucci R, Cremonesi P, Pignagnoli G (1999) A preliminary note on the use of enzymes in conservation: the removal of aged acrylic resin coatings with lipase. Stud Conserv 44(4):278-281 doi:10.2307/1506657

Beutel S, Klein K, Knobbe G, Königfeld P, Petersen K, Ulber R, Scheper T (2002) Controlled enzymatic removal of damaging casein layers on medieval wall paintings. Biotechnol Bioeng 80(1):13-21 doi:10.1002/bit.10341

Bosch-Roig P, Ranalli G (2014) The safety of biocleaning technologies for cultural heritage. Front Microbiol 5(155):doi: 10.3389/fmicb.2014.00155 doi:10.3389/fmicb.2014.00155

Bott G (1990) Amylase for starch removal from a set of 17 th century embroidered panels. The Conservator 14(1):23-29 doi:10.1080/01410096.1990.9995053

Božić N, Lončar N, Slavić Marinela Š, Vujčić Z (2017) Raw starch degrading $\alpha$-amylases: an unsolved riddle. Amylase 1(1):12 doi:10.1515/amylase-2017-0002 
Chapman V (1986) Amylase in a viscous medium-textile applications. The Conservator 10(1):711 doi:10.1080/01410096.1986.9995011

Cremonesi P (2013) Rigid gels and enzyme cleaning. In: Mecklenburg MF, Charola AE, Koestler RJ (eds) New insights into the cleaning of paintings: proceedings from the cleaning 2010 international conference. Smithsonian contributions to museum conservation. Universidad politecnica de Valencia and Museum Conservation Institute, Washington, DC, pp 179-183

Decoux S (2002) Enzymes used for adhesive removal in paper conservation: a literature review. J Soc Archivists 23(2):187-195 doi:10.1080/003379810220000006372

Ferrari M, Mazzoli R, Morales S, Fedi M, Liccioli L, Piccirillo A, Cavaleri T, Oliva C, Gallo P, Borla M, Cardinali M, Pessione E (2017) Enzymatic laundry for old clothes: immobilized alpha-amylase from Bacillus sp. for the biocleaning of an ancient Coptic tunic. Appl Microbiol Biot 101(18):7041-7052 doi:10.1007/s00253-017-8437-8

Germinario G, van der Werf ID, Palazzo G, Regidor Ros JL, Montes-Estelles RM, Sabbatini L (2017) Bioremoval of marker pen inks by exploiting lipase hydrolysis. Prog Org Coat 110:162-171 doi:https://doi.org/10.1016/j.porgcoat.2017.02.019

Gherardi F, Turyanska L, Ferrari E, Weston N, Fay MW, Colston BJ (2019) Immobilized enzymes on gold nanoparticles: from enhanced stability to cleaning of textile heritage. ACS Appl Bio Mat 2(11):5136-5143 doi:10.1021/acsabm.9b00802

Goodfellow BJ, Wilson RH (1990) A Fourier Transform IR study of the gelation of amylose and amylopectin. Biopolymers 30(13-14):1183-1189 doi:doi:10.1002/bip.360301304

Gupta R, Gigras P, Mohapatra H, Goswami VK, Chauhan B (2003) Microbial a-amylases: a biotechnological perspective. Process Biochem 38(11):1599-1616 doi:https://doi.org/10.1016/S0032-9592(03)00053-0

Htoon A, Shrestha AK, Flanagan BM, Lopez-Rubio A, Bird AR, Gilbert EP, Gidley MJ (2009) Effects of processing high amylose maize starches under controlled conditions on structural organisation and amylase digestibility. Carbohyd Polym 75(2):236-245 doi:https://doi.org/10.1016/j.carbpol.2008.06.016

Lopez-Rubio A, Flanagan BM, Shrestha AK, Gidley MJ, Gilbert EP (2008) Molecular rearrangement of starch during in vitro digestion: toward a better understanding of enzyme resistant starch formation in processed starches. Biomacromolecules 9(7):1951-1958 doi:10.1021/bm800213h

Luchese CL, Benelli P, Spada JC, Tessaro IC (2018) Impact of the starch source on the physicochemical properties and biodegradability of different starch-based films. J Appl Polym Sci 135(33):46564 doi:10.1002/app.46564

Mazzoli R, Giuffrida MG, Pessione E (2018) Back to the past-forever young: cutting-edge biochemical and microbiological tools for cultural heritage conservation. Appl Microbiol Biot 102(16):6815-6825 doi:10.1007/s00253-018-9121-3

Mazzuca C, Micheli L, Cervelli E, Basoli F, Cencetti C, Coviello T, lannuccelli S, Sotgiu S, Palleschi A (2014) Cleaning of paper artworks: development of an efficient gel-based material able to remove starch paste. ACS Appl Mater Inter 6(19):16519-16528 doi:10.1021/am504295n 
Mazzuca C, Poggi G, Bonelli N, Micheli L, Baglioni P, Palleschi A (2017) Innovative chemical gels meet enzymes: A smart combination for cleaning paper artworks. J Colloid Interf Sci 502:153-164 doi:https://doi.org/10.1016/j.jcis.2017.04.088

Palacios HR, Schwarz PB, D'Appolonia BL (2004) Effects of $\alpha$-amylases from different sources on the firming of concentrated wheat starch gels: relationship to bread staling. J Agr Food Chem 52(19):5987-5994 doi:10.1021/jf030384n

Palla F, Barresi G (2017) Biotechnology and conservation of cultural heritage. Springer International Publishing

Pereira C, Busani T, Branco LC, Joosten I, Sandu ICA (2013) Nondestructive characterization and enzyme cleaning of painted surfaces: assessment from the macro to nano level. Microsc Microanal 19(6):1632-1644 doi:10.1017/S1431927613013196

Ranalli G, Alfano G, Belli C, Lustrato G, Colombini MP, Bonaduce I, Zanardini E, Abbruscato P, Cappitelli F, Sorlini C (2005) Biotechnology applied to cultural heritage: biorestoration of frescoes using viable bacterial cells and enzymes. J Appl Microbiol 98(1):73-83 doi:10.1111/j.1365-2672.2004.02429.x

Schwarz I, Blüher A, Banik G, Thobois E, Maurer K-H (1999) The development of a ready-foruse poultice for local removal of starch paste by enzymatic action. Restaurator. vol 20, p 225

Sheldon RA (2007) Enzyme immobilization: the quest for optimum performance. Adv Synth Catal 349(8-9):1289-1307 doi:10.1002/adsc.200700082

Shibayama N, Eastop D (1996) Removal of flour paste residues from a painted banner with alpha-amylase. The Conservator 20(1):53-64 doi:10.1080/01410096.1996.9995103

Valentini F, Diamanti A, Carbone M, Bauer EM, Palleschi G (2012) New cleaning strategies based on carbon nanomaterials applied to the deteriorated marble surfaces: A comparative study with enzyme based treatments. Appl Surf Sci 258(16):5965-5980 doi:https://doi.org/10.1016/j.apsusc.2012.01.076

Valentini F, Diamanti A, Palleschi G (2010) New bio-cleaning strategies on porous building materials affected by biodeterioration event. Appl Surf Sci 256(22):6550-6563 doi:https://doi.org/10.1016/j.apsusc.2010.04.046

van der Maarel MJEC, van der Veen B, Uitdehaag JCM, Leemhuis H, Dijkhuizen L (2002) Properties and applications of starch-converting enzymes of the $\alpha$-amylase family. J Biotechnol 94(2):137-155 doi:https://doi.org/10.1016/S0168-1656(01)00407-2

van Soest JJG, Tournois H, de Wit D, Vliegenthart JFG (1995) Short-range structure in (partially) crystalline potato starch determined with attenuated total reflectance Fourier-transform IR spectroscopy. Carbohyd Res 279:201-214 doi:https://doi.org/10.1016/0008-6215(95)00270-7

Waterschoot J, Gomand SV, Fierens E, Delcour JA (2015) Production, structure, physicochemical and functional properties of maize, cassava, wheat, potato and rice starches. Starch - Stärke 67(1-2):14-29 doi:10.1002/star.201300238 


\section{Figure captions}

Fig. 1. FTIR spectra of silicon windows coated with (a) potato starch $2 \% \mathrm{w} / \mathrm{v}$ (P2) and (c) wheat starch $2 \% \mathrm{w} / \mathrm{v}$ (W2) and after $120 \mathrm{~min}$ and $240 \mathrm{~min}$ from the application of $\alpha$-amylase. Thermally aged silicon windows coated with P2 (b) and W2 (d) are also reported. (e) Summary of the activity in starch hydrolysis (SH) (\%) of $\alpha$-amylase after 120 and 240 min from the application on silicon wafers coated with potato and wheat starch $1 \% \mathrm{w} / \mathrm{v}$ (P1 and W1) and $2 \% \mathrm{w} / \mathrm{v}$ (P2 and W2) before and after accelerated thermal ageing.

Fig. 2. ATR-FTIR spectra of wool coated with thermally aged potato ( $a, c$ and e) and wheat (b, $\mathrm{d}$ and $\mathrm{f}$ ) starch $2 \% \mathrm{w} / \mathrm{v}$ and after $120 \mathrm{~min}$ from the application of $\alpha$-amylase by pipette (a and $b$ ), in gel ( $c$ and $d$ ) and on gel (e and f). Corresponding insets show the change in the absorbance of the peaks at 1022 and $1045 \mathrm{~cm}^{-1}$.

Fig. 3. ATR-FTIR spectra of wool coated with thermally aged potato $(a, c$ and e) and wheat (b, $\mathrm{d}$ and f) starch $5 \% \mathrm{w} / \mathrm{v}$ and after $120 \mathrm{~min}$ from the application of $\alpha$-amylase by pipette ( $a$ and $b$ ), in gel ( $c$ and $d$ ) and on gel (e and f). Corresponding insets show the change in the absorbance of the peaks at 1022 and $1045 \mathrm{~cm}^{-1}$.

Fig. 4. Secondary electron SEM images of wool coated with potato and wheat starch $5 \% \mathrm{w} / \mathrm{v}$ (P5 and W5) before and after the application of $\alpha$-amylase applied by pipette, in gel and on gel. Arrows indicate accumulation of starch.

Fig. 5. Photographic documentation, colorimetric characterization and microscopic observations of wool dyed with madder and coated with $2 \% \mathrm{w} / \mathrm{v}$ and $5 \% \mathrm{w} / \mathrm{v}$ potato (a-d) and wheat starch (e-h), before and after cleaning with $\alpha$-amylase applied by pipette, in gel and on gel. Results are separately reported according to the $L^{*}$ and $b^{*}$ colorimetric coordinates. Black dots are uncoated and unaged reference wool samples, while grey dots are aged starchcoated wool samples.

Fig. 6. Photographic documentation and colorimetric characterization of wool dyed with indigo ( $a$ and $b$ ) and saffron ( $c$ and $d$ ) and coated with $2 \% \mathrm{w} / \mathrm{v}$ and $5 \% \mathrm{w} / \mathrm{v}$ potato and wheat starch before and after cleaning with $\alpha$-amylase applied by pipette, in gel and applied on gel. Colorimetric data are reported as in Fig. 6. 
Fig. 1
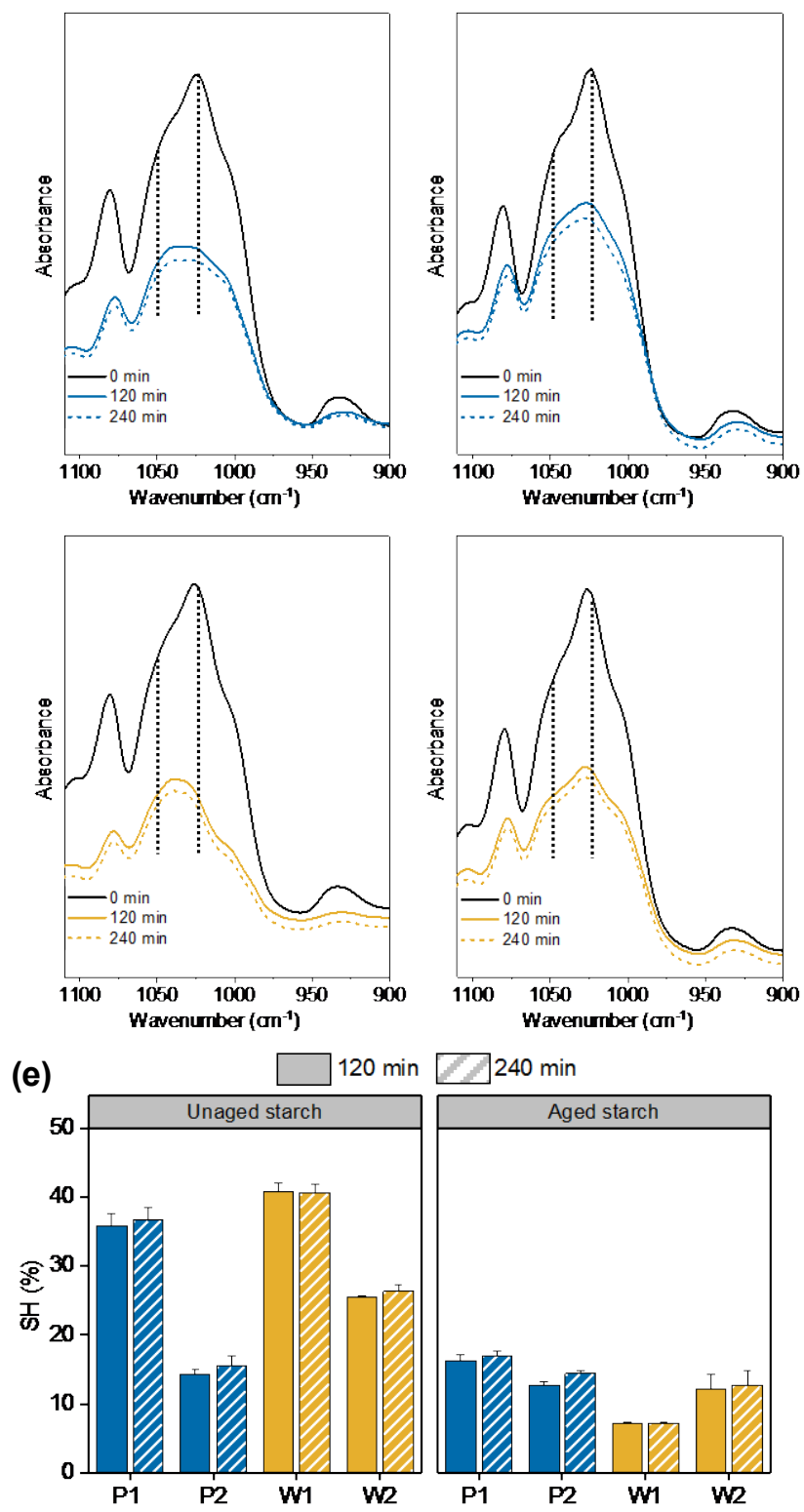
Fig. 2
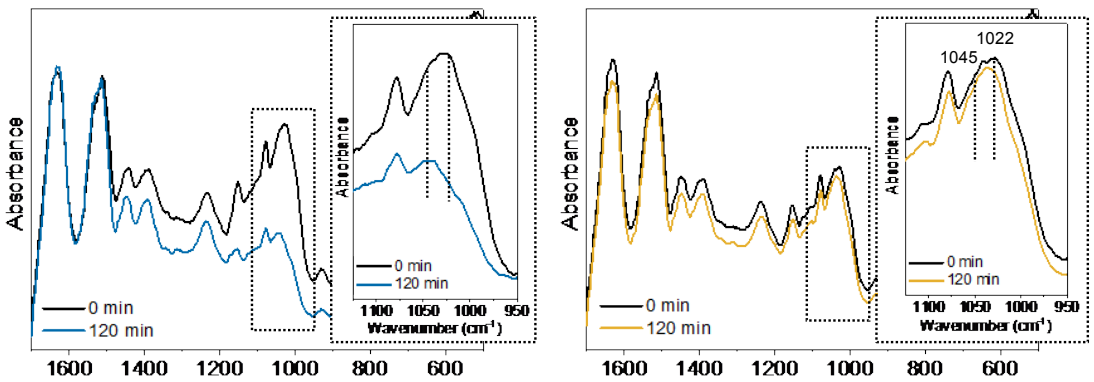

Wavenumber $\left(\mathrm{cm}^{-1}\right)$
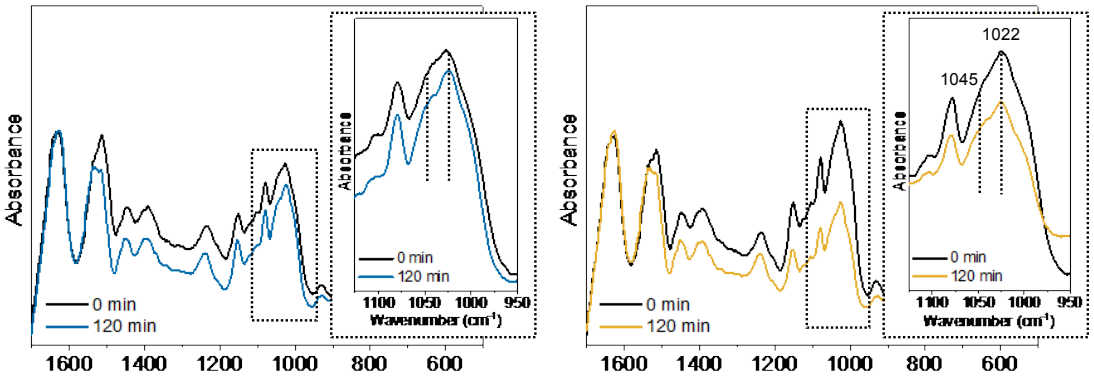

Waverumber $\left(\mathrm{cm}^{-1}\right)$
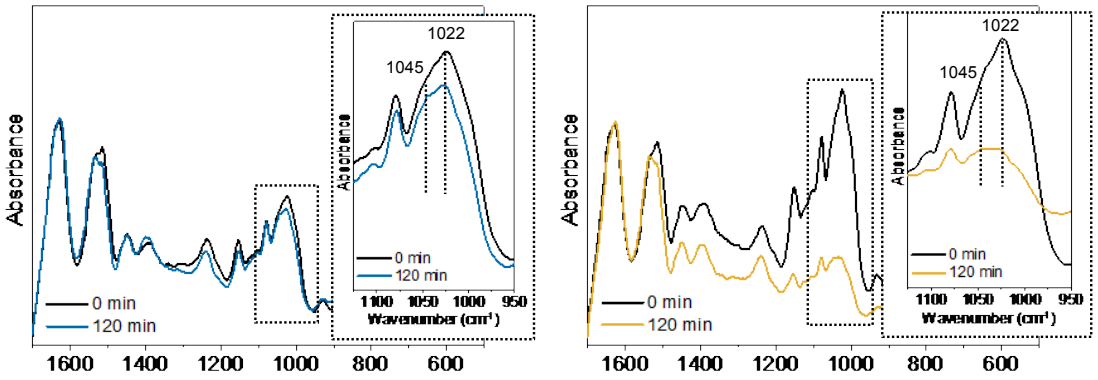

Waverumber (cm-1)

Waverumber $\left(\mathrm{cm}^{-1}\right.$ ) 
Fig. 3
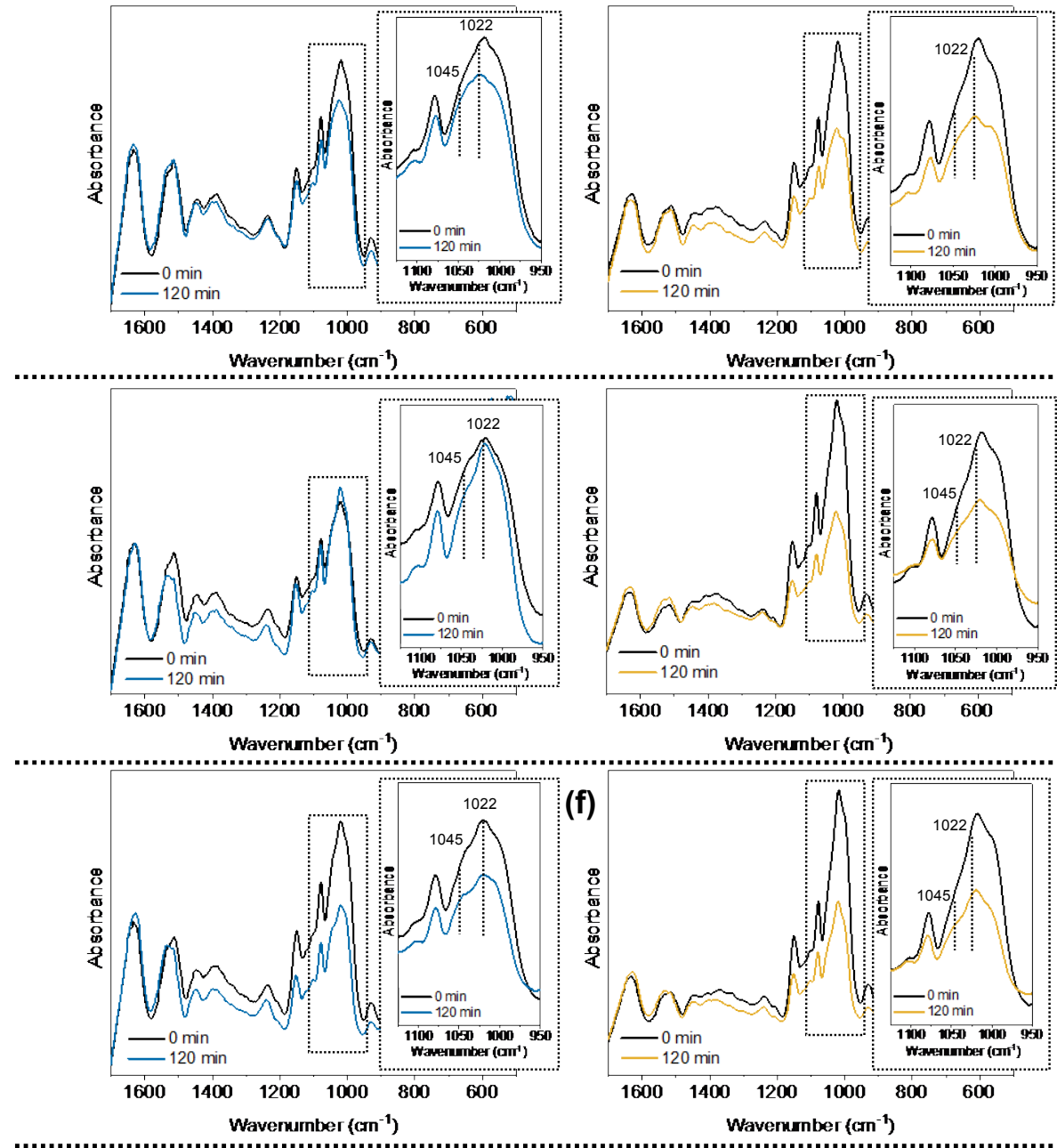
Fig. 4

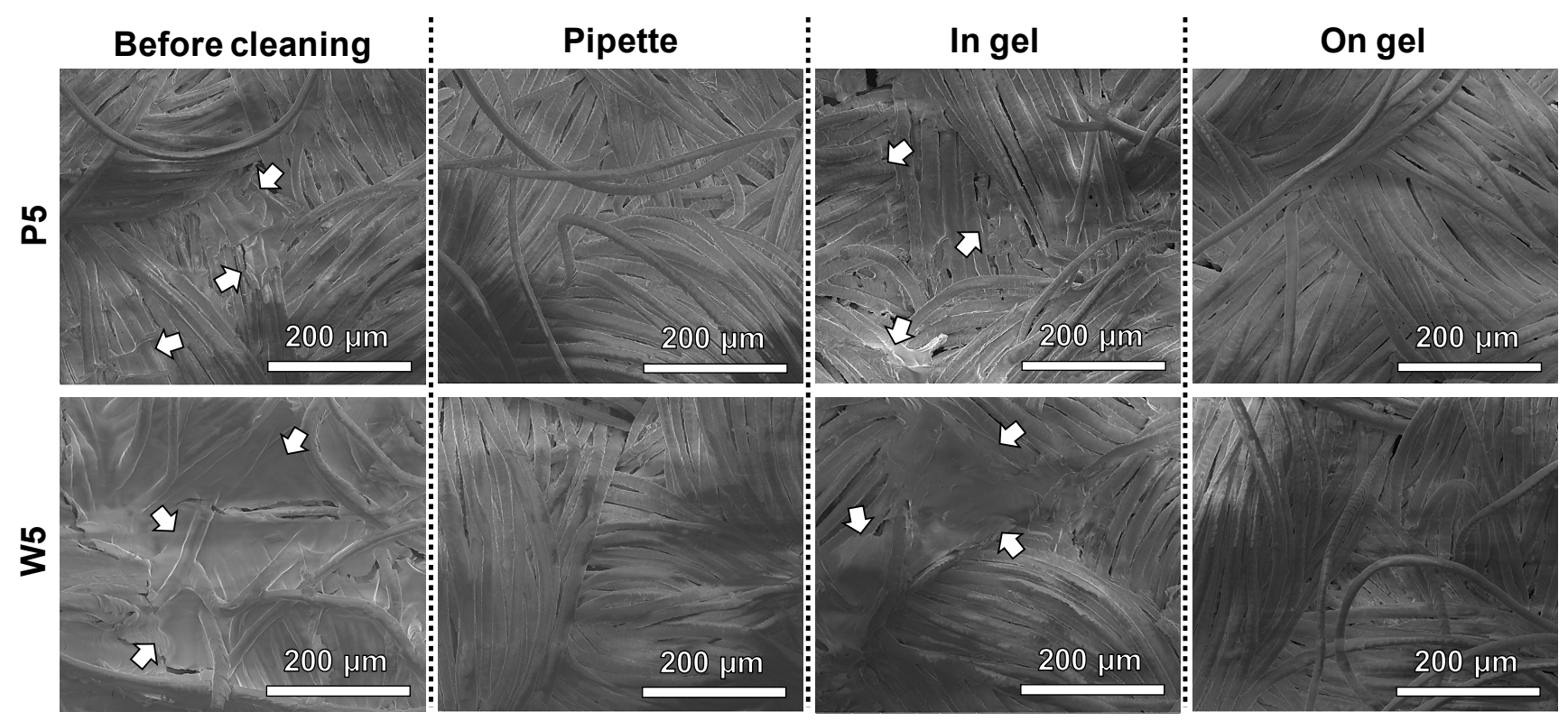


Fig. 5
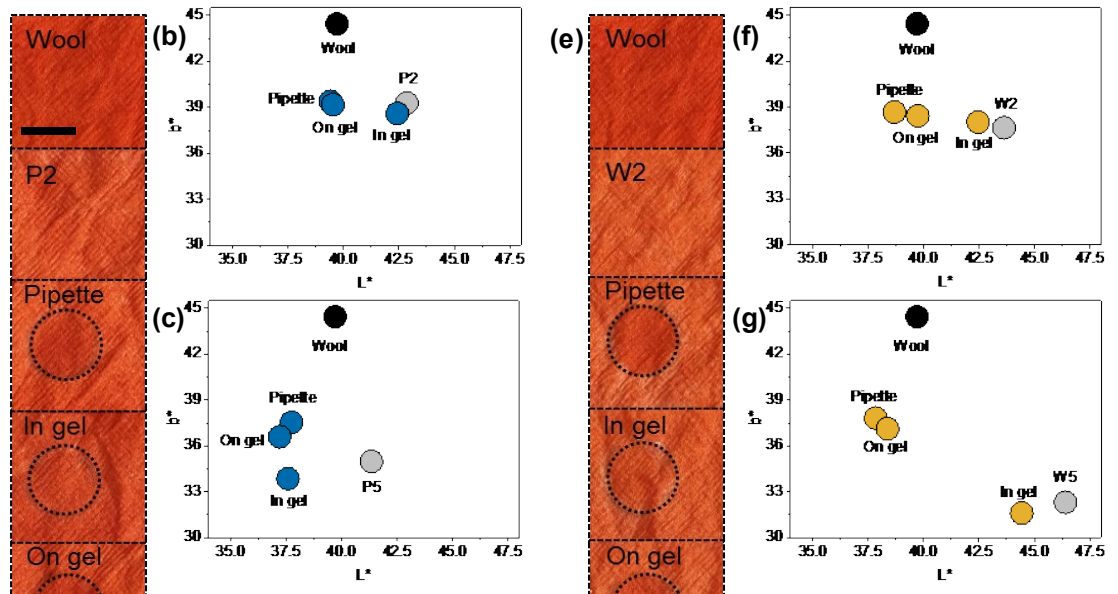

(d)
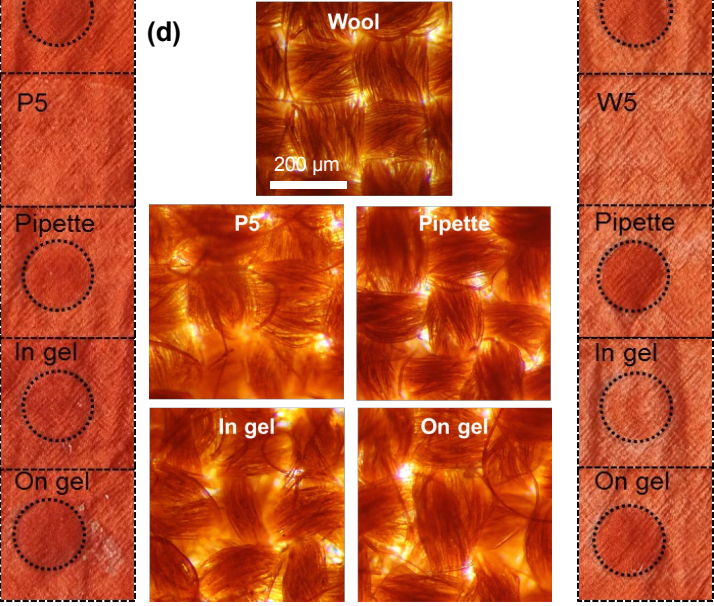

(h)
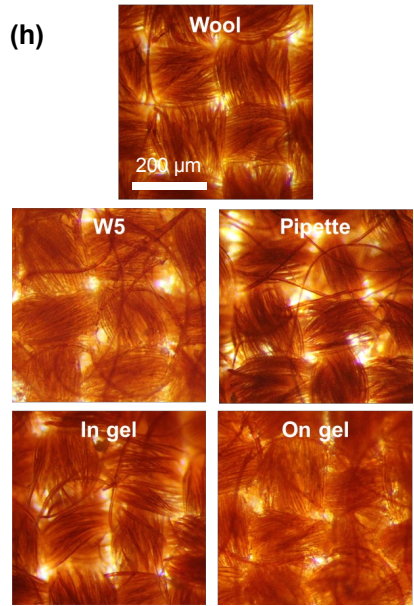
Fig. 6

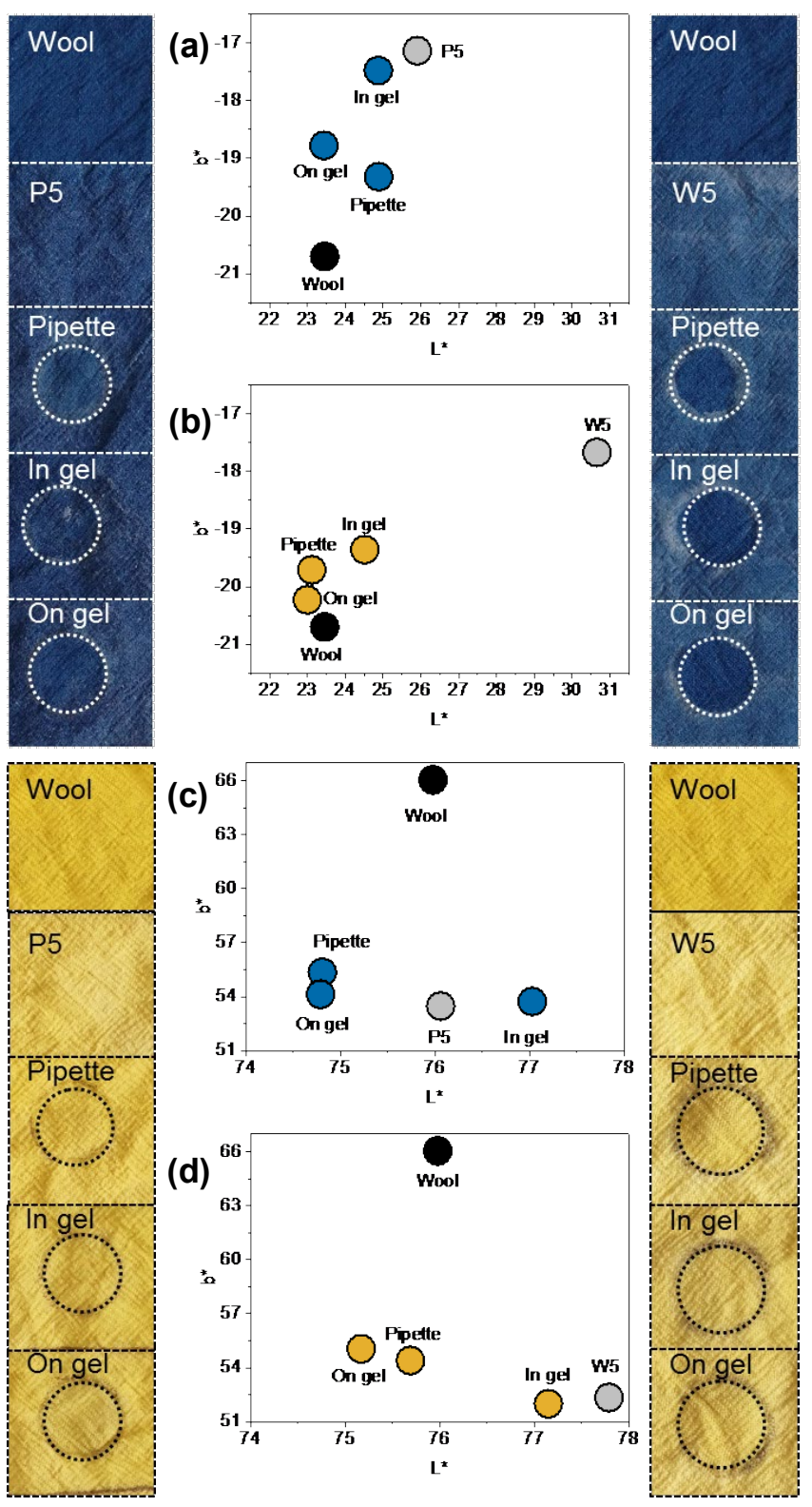

